\title{
Lait de chèvre en Europe
}

\section{Le lait de chèvre en Europe}

\author{
JC Le Jaouen, G Toussaint
}

Institut de l'élevage, 149, rue de Bercy, 75595 Paris, France

\begin{abstract}
Résumé - L'élevage caprin européen présente une forte spécialisation laitière : avec $3 \%$ du cheptel mondial, l'Europe produit $17 \%$ du lait de chèvre mondial. Les effectifs de la CEE ( 14,5 millions de têtes) sont en croissance depuis 10 ans, ils représentent $83 \%$ du cheptel total de l'Europe. La production du lait de chèvre est concentrée dans les pays du sud de l'Europe : la Grèce, l'Espagne et la France réalisent $86 \%$ de la production communautaire. Les systèmes de production évoluent de façon divergente : les élevages extensifs du sud et les petits troupeaux diminuent, les élevages laitiers spécialisés livrant leur lait à l'industrie se développent. Les systèmes de transformation à la ferme se modernisent. La transformation industrielle du lait est importante en France et en Espagne, dans les autres pays elle est peu développée. Le lait de chèvre est principalement transformé en fromages consommés de façon différente selon les pays. Les fromages de chèvre souffrent d'une insuffisance de protection de leurs dénominations traditionnelles. Le développement de la production de lait et de fromages de chèvre est directement lié aux marchés, mal connus dans certains pays, et qui restent essentiellement nationaux.
\end{abstract}

\section{lait de chèvre / Europe / systèmes d'élevage / transformation / fromages / évolution}

Summary - Goat's milk in Europe. European goat breeding has a strong dairy specialization; with $3 \%$ of the world goat population Europe produces $17 \%$ of the world goat milk. The number of goats within the EEC (14.5 million) has been increasing for the last 10 years. They represent $83 \%$ of the number of goats in Europe. The production of goat milk is mainly found in southern countries of Europe: Greece, Spain and France produce $86 \%$ of the milk in the EEC. The systems of production are different: the extensive breeding farms and the small flocks decrease. Specialized milk farms delivering their milk to industry are developing. The systems of transformation on the farm are getting more modern. The industrial transformation of the milk is important in France and in Spain but poorly developed in the other countries. Goat milk is mainly transformed into cheeses consumed differently in every country. Goat cheeses suffer from a lack of protection for their traditional denominations. The development of the production of milk and goat cheeses is directly linked to the markets which are not well-know in certain countries and which remain mainly national.

goat milk / Europe / breeding systems / transformation / cheeses / evolution 


\section{INTRODUCTION}

L'Europe possède une très ancienne tradition d'élevage de la chèvre dont témoignent la mythologie grecque et les nombreux sites archéologiques démontrant que la chèvre a été domestiquée depuis la préhistoire. De nos jours la chèvre et ses produits, notamment laitiers, constituent une réalité incontestable des économies agricoles modernes, avec toutefois des niveaux d'intégration variables selon les pays : les systèmes traditionnels de l'économie pastorale tournés vers l'autosubsistance y côtoient les élevages laitiers spécialisés associés à une infrastructure industrielle de transformation livrant des produits de large consommation. Ces contrastes constituent une réalité européenne hétérogène dont on peut néanmoins essayer de dégager les traits caractéristiques.

\section{L'EUROPE: ORIENTATION LAITIĖRE AFFIRMÉE}

Par rapport aux autres continents l'observation des données statistiques (tableau I) montre à l'évidence l'orientation laitière de l'élevage caprin européen qui produit $17 \%$ du lait de chèvre mondial avec seulement $3 \%$ de cheptel. À titre de comparaison, l'Amérique du Nord et centrale avec le même cheptel $(3 \%)$ n'assurent que $4 \%$ du lait et l'Asie, avec $59 \%$ du cheptel, ne produit que $53 \%$ du lait mondial. En revanche, la capacité européenne de viande caprine n'atteint que $4 \%$ du volume mondial, tandis que les pays en voie de développement totalisent $93 \%$ de la production mondiale (tableau I).

\section{UN CHEPTEL EN CROISSANCE DEPUIS 10 ANS}

L'entrée dans le Marché commun de l'Espagne et du Portugal a porté les effectifs de la CEE à 14,5 millions de caprins, soit une augmentation de $40 \%$. La communauté détient aujourd'hui $83 \%$ du cheptel caprin européen (hors ancienne URSS).

Avec $84 \%$ du cheptel de la CEE, les pays méditerranéens représentent la part la plus importante des effectifs, dont $38 \%$ en Grèce, $23 \%$ en Espagne, I'ltalie et la France possédant chacune $9 \%$. Cette répartition géographique est résumée sur la figure 1.

Depuis 10 ans, le troupeau caprin européen a progressé de $30 \%$, mais la croissance annuelle s'est légèrement ralentie d'après les dernières estimations.

On distingue, selon les pays, des évolutions différentes :

- dans un seul, la France, le cheptel caprin a régressé, compensé par un accroissement de productivité laitière ;

- les effectifs au Portugal (depuis 1986), en Grèce, en Italie et en Belgique sont en forte augmentation ; en Allemagne également, du fait de la prise en compte des caprins de l'ex-RDA depuis 1989;

- la progression reste très forte en Espagne (depuis 1986), au Royaume-Uni et aux Pays-Bas ; dans ces pays les cheptels ont plus que doublé.

La dernière enquête sur les structures (1987) réalisée par la CEE rapporte que 703600 exploitations agricoles, soit $8 \%$, détenaient 10 millions de caprins, $4 \%$ de ces exploitations étaient spécialisées en production caprine, $13 \%$ étaient en horssol et seulement $5 \%$ d'entre elles dépassaient 50 ha de surface agricole utile. 


\section{LE LAIT DE CHÈVRE EST LOCALISÉ AU SUD DE L'EUROPE}

Avec un potentiel annuel de production communautaire estimé à 2000 millions de litres de lait de chèvre, le classement des 4 principaux pays producteurs s'établit dans l'ordre suivant: Grèce : $30 \%$ de la production laitière (avec $46 \%$ du cheptel) ; France : $28 \%$ de la production laitière (avec $9 \%$ du cheptel); Espagne : $28 \%$ de la production laitière avec $28 \%$ du cheptel ;
Italie : $8 \%$ de la production laitière (avec $9 \%$ du cheptel). Ainsi, 3 pays, la Grèce, la France et l'Espagne assurent globalement $86 \%$ de la production communautaire de lait de chèvre (fig 2).

\section{DES SYSTĖMES DE PRODUCTION DIVERSIFIÉS}

Une synthèse des observations sur les orientations technico-économiques permet

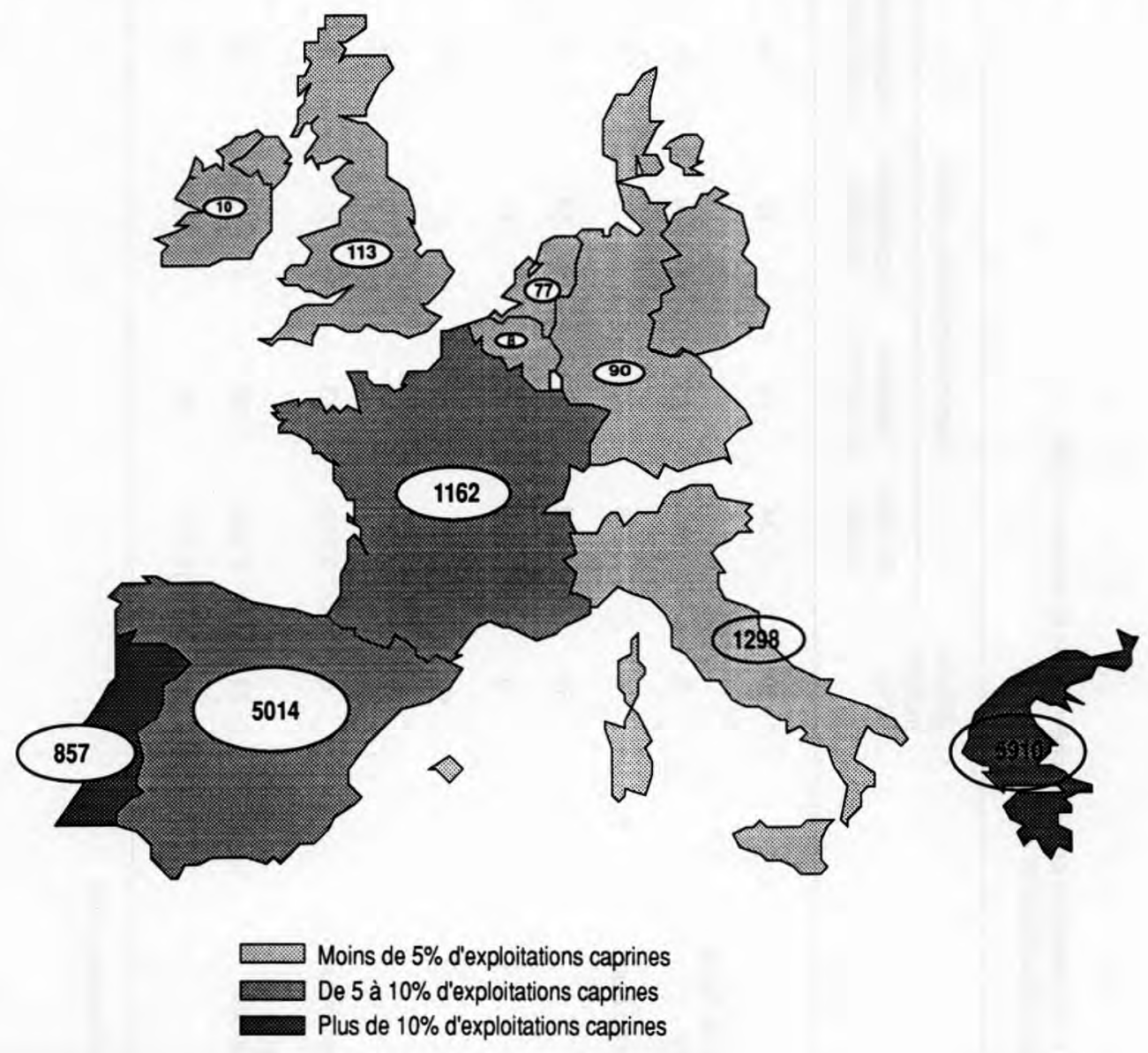

Fig 1. Les effectifs caprins dans la CEE en 1990 (milliers de têtes).

Goat populations in the EEC in 1990. 
Tableau I. La production de lait de chèvre dans le monde en 1991.

World goat milk production in 1991.

\begin{tabular}{|c|c|c|c|c|c|c|c|}
\hline & \multirow{2}{*}{$\begin{array}{c}\text { Effectif } \\
\text { mondial } \\
\text { (\%) }\end{array}$} & \multicolumn{4}{|c|}{ Production de lait de chèvre } & \multicolumn{2}{|c|}{ Consommation kg/hab/an (1989) } \\
\hline & & $\begin{array}{l}\text { En } 1000 \\
\text { tonnes }\end{array}$ & $\begin{array}{c}\text { Variation } \\
1971 / 91 \text { (\%) }\end{array}$ & $\begin{array}{l}\% \text { production } \\
\text { mondiale }\end{array}$ & $\begin{array}{l}\% \text { production } \\
\text { lait de vache }\end{array}$ & $\begin{array}{l}\text { Lait de } \\
\text { chèvre }\end{array}$ & $\begin{array}{l}\text { Lait de } \\
\text { vache }\end{array}$ \\
\hline Afrique & 30 & 2134 & 16 & 21 & 14 & 3,263 & 16,524 \\
\hline $\begin{array}{l}\text { Amérique du Nord } \\
\text { et centrale }\end{array}$ & 3 & 399 & 25 & 4 & $<1$ & 0,922 & 172,773 \\
\hline Amérique du Sud & 4 & 178 & 33 & 2 & 1 & 0,526 & 80,377 \\
\hline Asie & 59 & 5392 & 34 & 53 & 10 & 1,265 & 10,948 \\
\hline Europe & 3 & 1785 & 13 & 17 & 1 & 3,521 & 349,605 \\
\hline Océanie & $<1$ & - & - & - & $<1$ & - & 469,338 \\
\hline URSS & 1 & 350 & 16 & 3 & $<1$ & 1,261 & 321,385 \\
\hline Pays développés & 6 & 2153 & 13 & 21 & 1 & 1,723 & 283,169 \\
\hline $\begin{array}{l}\text { Pays en voie de } \\
\text { développement }\end{array}$ & 94 & 8083 & 28 & 79 & 8 & 1,623 & 17,003 \\
\hline Monde & 100 & 10237 & 25 & 100 & 2 & 1,647 & 80,585 \\
\hline
\end{tabular}

Source : annuaire de la FAO. 


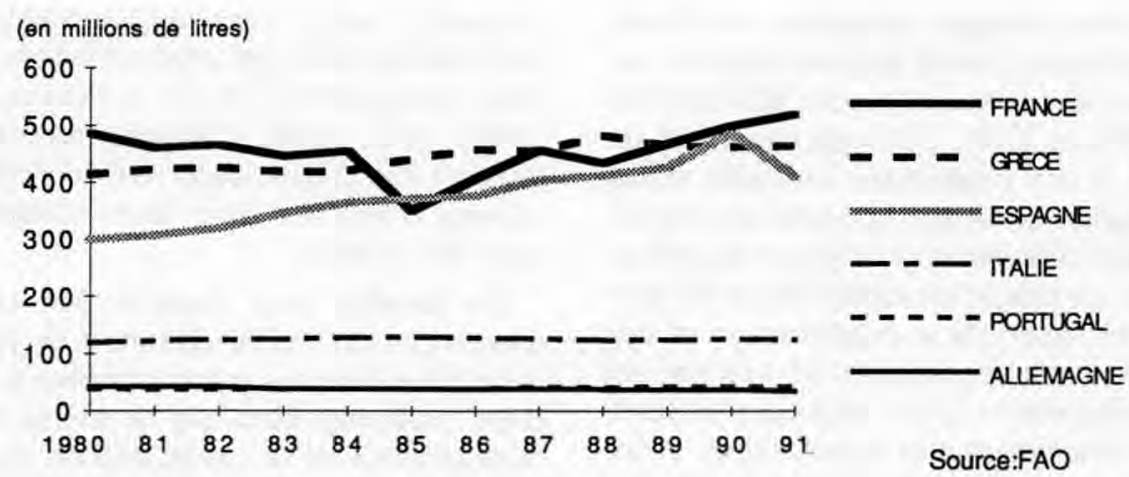

Fig 2. Production de lait de chèvre dans la CEE. Goat milk production in the EEC.

d'établir une typologie sommaire des systèmes de production contribuant au potentiel de production européen.

On distingue 3 systèmes de base.

Le premier est extensif, surtout lié aux zones de végétation arbustive méditerranéenne et aux pâturages de montagne, la taille des troupeaux se révèle plus ou moins importante (quelques dizaines à plusieurs centaines de têtes) selon que les caprins se trouvent incorporés ou non à des troupeaux ovins transhumants ou nomades. La spécialisation reste rare et les capitaux engagés faibles. Les populations caprines exploitées localement sont bien adaptées à ce mode d'élevage. Peu productives en lait (70 à 150 V/an), les chèvres donnent en moyenne chacune un chevreau pour la boucherie. Les éleveurs d'un faible niveau technique exercent parfois cette activité à temps partiel. Ce système est globalement en régression constante à cause des contraintes sociales et économiques (régions défavorisées).

Présent dans toute l'Europe et mêlant des modes l'élevage semi-intensifs et intensifs sur de très petites surfaces, le second système de production a pour principale raison d'être l'auto-consommation familiale avec de petits effectifs (inférieurs à 10-20 têtes). II se révèle donc peu exigeant en capital. Les races laitières exploitées sont plus productives. Le niveau technique des éleveurs est variable, sauf dans le cas du modèle du hobby anglo-saxon où la passion pour l'animal s'accompagne d'un intérêt technique soutenu. Le temps de travail demandé par cette occupation est forcément limité. Ce système de production stagne dans les pays où il est considéré comme un loisir, il régresse de façon continue dans ceux où il se trouve lié à la densité de la population rurale.

Lorsque l'objectif de la production caprine est la vente de lait ou la transformation et la vente de fromages, le système d'élevage devient intensif et tend vers la spécialisation. Les races laitières se sélectionnent sur leur productivité et le niveau technique des éleveurs progresse car ils bénéficient en général d'un encadrement technique. On distingue cependant 2 soussystèmes.

L'un est fondé sur la vente du lait à des transformateurs industriels. Afin de rémunérer la main-d'œuvre généralement familiale; l'exploitant s'oriente vers des unités de grande taille et tend à se spécialiser. 
Dans les élevages spécialisés de l'ouest de la France, l'unité de main-d'œuvre exploite 142 chèvres et produit 103000 I de lait (724 I/chèvre). L'élevage caprin est associé à des productions agricoles moins exigeantes en temps de travail, comme les cultures céréalières et la viande bovine ou ovine. Le besoin en capital devient important en raison de la mécanisation et des équipements nécessaires. Ce système est en progression pour plusieurs raisons, liées notamment à la diversification imposée par l'application des quotas à la production de lait de vache et au maintien du revenu agricole.

L'autre sous-système est fondé sur la fabrication à la ferme de produits laitiers caprins destinés à la vente; les troupeaux se révèlent alors de moins grande taille, car au besoin en main-d'œuvre demandé par l'élevage, s'ajoutent ceux de la fabrication fromagère et de la vente des fromages. Dès qu'elle n'est plus marginale, cette activité devient très spécialisée. La surface nécessaire est plus faible, et le "hors-sol» tend à se développer. L'engagement en capitaux reste modeste. Ce système de production stagne en raison de sa très forte contrainte en maind'œuvre.

L'évolution du mode de commercialisation vers la production industrielle peut être considérée comme un moyen de mesurer les progrès accomplis dans chaque pays membre pour constituer une filière lait de chèvre structurée.

\section{TRANSFORMATION : DU PETIT ARTISANAT À LA GRANDE INDUSTRIE}

L'analyse des systèmes de transformation du lait de chèvre en Europe montre une grande hétérogénéité de situations en fonction de chaque histoire nationale de la production laitière caprine, des traditions de consommation des produits laitiers caprins conjuguées avec les évolutions récentes, et du niveau d'intégration du secteur lait de chèvre dans les économies laitières modernes avec leurs infrastructures industrielles.

Un parallèle peut d'ailleurs être établi avec la situation du lait de brebis, en particulier dans les pays méditerranéens à tradition pastorale, bien que le niveau d'industrialisation de la transformation du lait de brebis y soit plus développé que celui du lait de chèvre en raison de plus fortes habitudes de consommation des fromages de brebis.

II est remarquable d'observer que dans tous les pays coexiste une transformation à la ferme généralement orientée vers la vente locale, avec une transformation industrielle plus ou moins développée. Or, du fait de l'urbanisation croissante, les marchés de consommation se concentrent de plus en plus dans les villes dont l'approvisionnement implique : un regroupement de l'offre en volumes suffisants, une régularité des livraisons tant en quantité qu'en qualité, un allongement des circuits de distribution, la possibilité d'accès aux formes modernes de distribution et une organisation commerciale en conséquence.

Face à ces exigences, la transformation sous des formes traditionnelles en petites structures fermières ou artisanales se révèle souvent inadaptée car ne disposant pas des volumes suffisants, ni des moyens technologiques et structures commerciales nécessaires.

De plus, la taille restreinte des ateliers et leur atomisation ne permettent pas de dégager des moyens de recherchedéveloppement d'origine professionnelle ce qui explique que, dans la plupart des pays, la recherche-expérimentation dans le secteur laitier caprin dépende largement des financements publics, lesquels sont 
généralement limités pour le lait de chèvre. Seuls les pays possédant une filière laitière caprine structurée ou des groupes industriels importants s'intéressant au lait de chèvre disposent aujourd'hui des ressources financières et des capacités scientifiques pour conduire des programmes de recherche-développement.

La figure 3 présente, à partir des données disponibles, les pourcentages des transformations industrielles et artisanales (ou fermières) du lait de chèvre.

Trois groupes de pays se distinguent.

Les pays dont la majeure partie du lait est transformée industriellement sont: 1) la France avec $58 \%$ du lait, 260 millions de litres collectés annuellement par une centaine d'entreprises, dont 5 traitent la moitié de la production industrielle, 2 groupes dépassant 50 millions de litres par an ; quelques groupes de dimension internationale ont une activité lait de chèvre; 2) l'Espagne : $65 \%$ du lait sont collectés par des laiteries industrielles souvent de petite taille ; 3) les Pays-Bas et la Norvège, avec des volumes de collecte industrielle beaucoup plus faibles (moins de $\mathbf{2 0}$ millions de litres par an).
Le deuxième groupe comporte les pays présentant une transformation industrielle minoritaire coexistant avant une transformation artisanale traditionnelle prépondérante: Italie, Grèce et, pour de faible volumes, le Royaume-Uni.

Les autres pays, où il n'existe pas de filière industrielle spécialisée significative, sont l'Allemagne, le Portugal, la Belgique, etc...

Parallèlement au développement du système industriel, la petite transformation artisanale peut se révéler performante et s'adapter aux impératifs des marchés modernes.

Des ateliers fromagers généralement spécialisés dans les fromages traditionnels avec une gamme de produits se sont développés avec succès dans plusieurs pays: France, Espagne, Italie, Pays-Bas, Royaume-Uni. II s'agit d'un mode de transformation capable d'être compétitif face au système industriel et susceptible de s'adapter aux formes modernes de distribution.

Un des freins de l'industrialisation de la transformation a longtemps été la saisonnalité de la production du lait de chèvre.

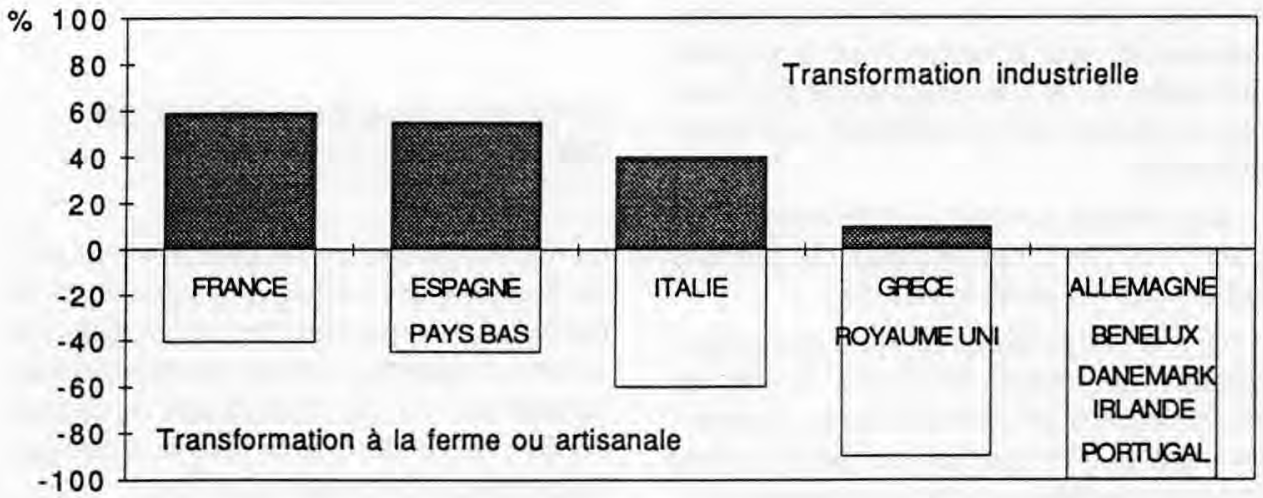

Fig 3. Estimation des parts de transformation de la production de lait de chèvre par pays. Percentage of goat milk transformation in each country. 
Aujourd'hui, même si l'irrégularité saisonnière de la collecte continue de poser des problèmes, des solutions existent, soit au niveau de la production (en PoitouCharentes le rapport des volumes de lait collectés en hiver et en été est passé de 1 pour 6 à 1 pour 3 en 10 ans), soit au niveau des produits, grâce aux technologies de report (caillé et lait concentré congelé pour les pâtes lactiques, report sous vide pour les pâtes pressées).

\section{LA CONSOMMATION DES PRODUITS LAITIERS CAPRINS}

En dehors de la France, il existe peu d'études sur le comportement des consommateurs européens face aux produits caprins ; quelques-unes seulement intègrent les fromages de chèvre. L'Europe est constituée d'un ensemble de pays dans lesquels les fromages font partie des habitudes de consommation, en moyenne $17 \mathrm{~kg}$ par habitant et par an dans la CEE. Cependant, la quantité moyenne de fromages de chèvre consommée reste inférieur à $500 \mathrm{~g}$, variant de $4 \mathrm{~kg}$ à quelques dizaines de grammes par habitant selon les pays. Cela met également en évidence sa forte consommation traditionnelle dans les pays du sud. Toutefois il est à regretter que certains de ces résultats ne prennent pas en compte les fromages de fabrication artisanale.

Ce constat conduit à différencier l'approche de la consommation de produits laitiers caprins selon la latitude :

- dans les pays situés au nord d'une ligne passant au niveau de Paris, le lait de chèvre sera plutôt consommé en nature ; cela explique le maintien de petites unités caprines destinées à l'auto-consommation familiale; le fromage se consomme moins couramment, plutôt frais, au petit déjeuner ou dans des sandwichs à midi en Alle- magne, Danemark, Irlande, Pays-Bas et Royaume-Uni ; la dégustation de fromages pendant le repas du soir se développe en Allemagne et en Belgique ;

- en France et dans les pays du sud, la consommation de lait liquide est marginale, l'essentiel de la production laitière est transformée en fromages; ils se consomment à la fin du repas en France, en ingrédient de cuisine ou au repas du soir en Italie, en accompagnement de plats traditionnels et au moment de l'apéritif en Espagne et au Portugal, au petit déjeuner et dans les salades en Grèce.

Une tendance générale vers des goûts plus neutres est observée chez la plupart des consommateurs européens. Cela va à l'encontre de certains types de fabrications à goût marqué du sud de l'Europe, et favorise les fromages frais ou peu affinés, en particulier les pâtes fraîches stabilisées lancées récemment par des groupes industriels.

Les quelques études de comportement des consommateurs montrent que, dans les grands centres de consommation, le fromage de chèvre est assez peu connu des ménages modernes, sauf en France. II est considéré comme un produit au goût prononcé et de prix élevé.

\section{IDENTIFICATION ET PROTECTION DES PRODUITS}

La majeure partie du lait de chèvre produit en Europe étant destiné à la fabrication de fromages, la protection de ces produits est un enjeu essentiel de leur développement commercial, et par conséquent de la production de lait de chèvre face à la concurrence des produits laitiers bovins.

Un recensement des fromages de chèvre et de brebis a été entrepris au niveau mondial par la Fédération internatio- 
nale de laiterie (groupe A 7) afin d'identifier pour chaque pays les principales variétés. Plus de 200 variétés de fromages fabriqués avec du lait de chèvre ont été recensées, dont une centaine pour l'Europe.

Cette étude a cependant conduit aux constats suivants:

- à l'exception de la France, très peu de fromages sont identifiés comme étant fabriqués au seul lait de chèvre ; en particulier, la tradition méditerranéenne qui consiste à mélanger les laits de chèvre, de brebis et de vache est source de confusion ;

- les dénominations génériques ou les dénominations de spécialités régionales sont rarement définies dans les réglementations nationales ;

- enfin, seule la France possède des appellations d'origine spécifiques aux fromages de chèvre (6 appellations).

II apparaît donc clairement qu'une des conditions du développement du marché des produits laitiers caprins en Europe est liée à une meilleure protection et une meilleure identification des produits. Cela concerne aussi bien les produits régionaux traditionnels que les nouveaux produits lancés récemment par des groupes industriels dans le cadre d'une politique de diversification de leur gamme afin de s'adapter aux évolutions de la consommation.

\section{CONCLUSION}

Les évolutions récentes montrent que la production laitière caprine s'intègre de plus en plus dans le processus général de modernisation de la filière laitière européenne.

Dans le contexte communautaire d'excédents des produits laitiers, le secteur caprin, comme celui du lait de brebis, doit se définir une stratégie de croissance en adéquation avec ses débouchés qui demeurent essentiellement nationaux.

La production de lait de chèvre n'étant pas soumise à la politique de limitation de la production mise en place pour le lait de vache, il existe un risque réel de développement anarchique générateur d'excédents risquant de perturber les marchés existants.

Pour les régions traditionnellement productrices de lait de chèvre (sud de l'Europe), la crainte d'une délocalisation des zones d'élevage constitue une préoccupation accentuée par la création du marché unique.

Enfin, outre les fromages traditionnels qui doivent être mieux protégés, le développement du secteur laitier caprin passe par une diversification de ses produits lui permettant d'élargir ses marchés de consommation en s'adaptant aux nouveaux goûts. 